\title{
Investigation of the Unsteady Behavior of a Circulation Control Wing Using Computational Fluid Dynamics
}

\author{
Jonathan A. Lichtwardt ${ }^{*}$ and David D. Marshall ${ }^{\dagger}$ \\ California Polytechnic State University, San Luis Obispo, CA, 93407-0352
}

\begin{abstract}
This paper details the results of an investigation into the unsteady behavior of a circulation control wing using computational fluid dynamics. Oscillations in the lift coefficient of up to $10 \%$ are observed for steady state simulations. An investigation into the source of the unsteadiness is underway, and the results to date are presented. It is shown that the periodic oscillations are independent of the above the wing mounted engine effects on the cruise efficient short take-off and landing aircraft. The oscillations are also a viscous phenomenon that does not dampen as the solution marches through steady state. It is proposed that the cause of the oscillations is due to high streamline curvature at the trailing edge inboard wing section, due to flow turning caused by the slot flow normal condition at the circulation control slots. This paper presents the results into the origin of this unsteadiness.
\end{abstract}

\section{Nomenclature}

$C_{L} \quad=$ coefficient of lift

$C_{p} \quad=$ coefficient of pressure

$C_{P}{ }^{*} \quad=$ jet stall

$C \quad=$ chord length

$k \quad=\quad$ turbulent kinetic energy

$x=$ spanwise coordinate direction

$y^{+} \quad=$ dimensionless wall dimension

$\mathrm{Z}=$ chordwise coordinate direction

$\omega \quad=$ specific dissipation rate

\section{Introduction}

$\mathrm{U}$ NSTEADY behavior of circulation control wings (CCW) has been presented in several works in the literature. They are classified here as either physical or induced. Abramson and Rogers ${ }^{1}$ present the two most noteworthy types of physical unsteady behavior, categorized as forms of jet stall of a circulation control airfoil. The first type is called jet detachment stall; it is characterized by premature separation of the Coanda jet from the surface. The result is an immediate loss in suction on the Coanda surface and a sharp loss in lift. The second type of jet stall is called $C_{P}{ }^{*}$ stall. This type of stall creates an unsteady wake region induced by critical pressure on the Coanda surface; it is not typified by separation. $C_{P}{ }^{*}$ stall has also been described as the result of a breakdown in the shear layer formed between the high speed jet flow and freestream flows ${ }^{2}$. The second class of unsteady behavior is discussed in Liu ${ }^{3}$. Liu focused on unsteady behavior of a circulation control airfoil through pulsed jet flow and separation induced through angle of attack. Although there are similarities that can be drawn from the above phenomena, they are distinctly different from the unsteadiness that is the topic of this investigation.

\section{A. Advanced Model for Extreme Lift and Improved Aeroacoustics (AMELIA)}

The Advanced Model for Extreme Lift and Improved Aeroacoustics, or AMELIA, is the basis for this work. AMELIA, shown in Fig. 1, is the result of a current research program, led by Cal Poly, which is funded under NASA's Subsonic Fixed Wing Program, responsible for The Integrated Modeling and Verification of Hybrid WingBody Low Noise ESTOL Aircraft. One of the primary purposes of this contract is to develop and validate predictive

\footnotetext{
${ }^{*}$ Graduate Student, Aerospace Engineering Department, Student Member AIAA.

${ }^{\dagger}$ Associate Professor, Aerospace Engineering Department, Senior Member AIAA.
} 
modeling capabilities for AMELIA (as well as other $\mathrm{N}+2$ generation aircraft), in both aerodynamic and acoustic disciplines. The contract is comprised of two phases. The first phase was composed of selecting the design of a powered lift N+2 generation Cruise Efficient, Short Take-Off and Landing (CESTOL) aircraft. The second phase of the project includes the detailed design, manufacturing, and testing of a wind tunnel model based on the selected CESTOL concept. The project culminates in a large scale wind tunnel test to be carried out in the 40 foot $\mathrm{x} 80$ foot wind tunnel at the National Full-Scale Aerodynamic Complex (NFAC) at NASA Ames Research Center, in the fall of 2011. The wind tunnel model is a $1 / 11^{\text {th }}$ scale, with a 10 foot wing span, incorporating a circulation control wing and turbofan propulsion simulators (TPS). Finally, an open database with data collected from the wind tunnel test will be created and released to the general research community to further improve the modeling capabilities of circulation control flows. Marshall and Jameson ${ }^{4}$ give a complete description of all aspects of the AMELIA

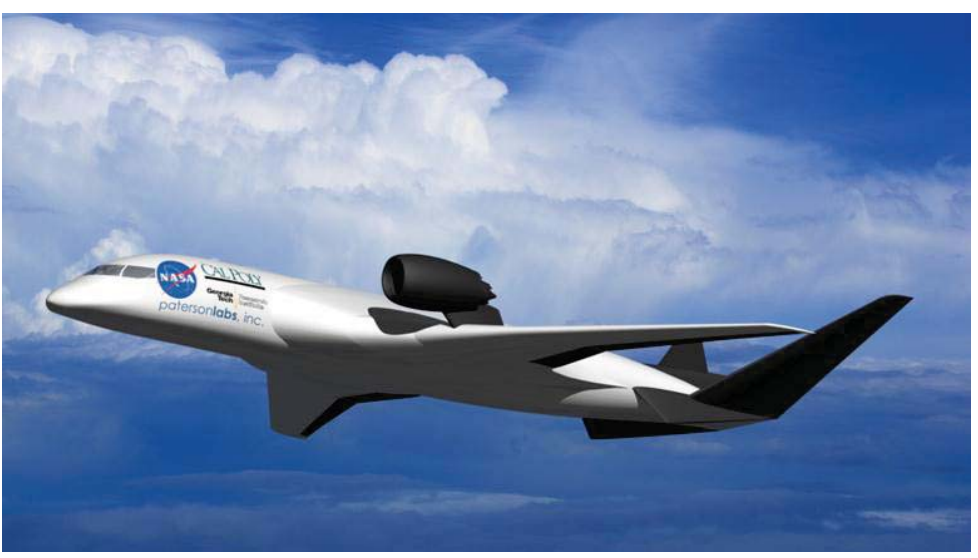

Figure 1. AMELIA, an N+2 generation CESTOL aircraft for use in Cal Poly's future wind tunnel test. project.

As mentioned, one of the primary purposes of this contract is to develop and validate predictive modeling capabilities for AMELIA. Imperative to this is accurate modeling of the complex flow physics - in particular, jet flow over the Coanda surface, shear layer mixing, and the coupling of engine exhaust with circulation control jet flow $^{5}$. Figure 2 shows the trailing edge slot and flap geometry. Unlike many early CCW designs ${ }^{6}$, which

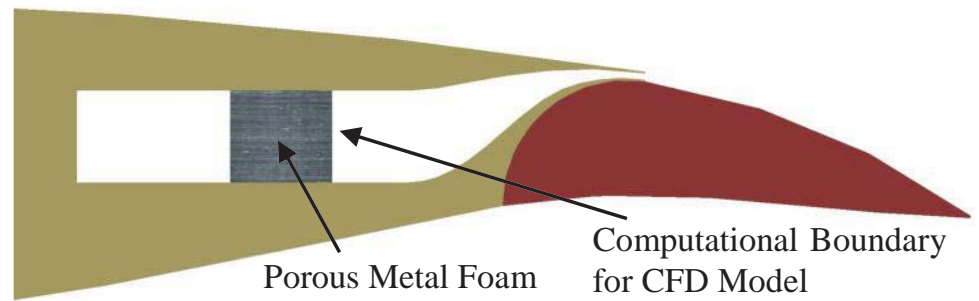

Figure 2. Cross section of the trailing edge circulation control slot and dual radius flap. incorporated rounded trailing edge airfoils, this circulation control system utilizes a sharp trailing edge with a dual radius flap. The flap has been shown to keep the flow attached longer over the Coanda surface, resulting in less momentum loss ${ }^{7}$. It should be noted there is a similar slot located on the leading edge of the wing. In Fig. 2, the edge of the metal foam (used to straighten the flow) is the computational plane for the CFD model. This is a convenient plane because the pressure is known.

\section{B. Objective}

This research was initiated as a part of an earlier effort to find the optimal placement of the engine (with respect to both chordwise and vertical directions), in order to maximize engine exhaust entrainment and the associated increase in lift in the low speed configuration ${ }^{8}$. The figure of merit for the optimum location was the traditional aerodynamic metric of lift to drag ratio, however difficulty in attaining a true "steady-state" solution made it such that the engine entrainment performance metric had to be based upon the angle of the thrust deflection. Therefore, the objective of this paper is to provide an understanding of the periodic unsteadiness noticed in the lift coefficient force monitor for steady state CFD solutions. Similar oscillations have been reported in several other works ${ }^{5}$ and thus an understanding of the behavior is in need.

\section{Model Description}

The following section outlines the general model description for both the experimental and computational efforts of this project.

\section{A. Experimental Model}

The experimental aspect of this contract culminates in a full scale wind tunnel test of AMELIA. Although this paper is focused on the computational modeling of AMELIA, it is important to highlight several features of the 
model as they define computational boundaries and the like. An in depth description of the experimental effort of this project is provided in Jameson et al. ${ }^{9,10}$. Figure 3 shows a cut-away view of the wind tunnel model and internals, highlighting the leading and trailing edge circulation control slots, and TPS units.

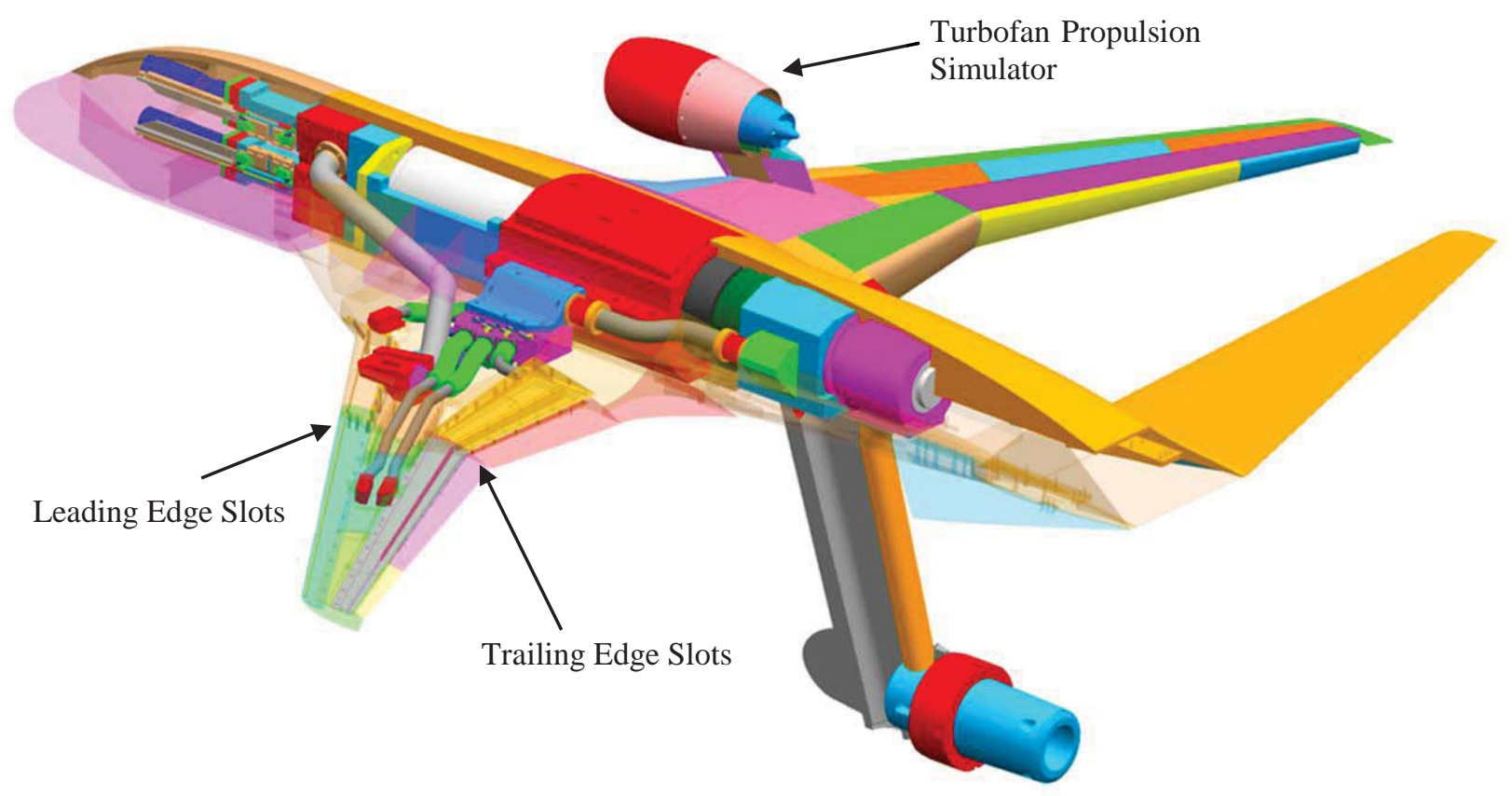

Figure 3. Detail view of AMELIA revealing internal components.

\section{B. Computational Model}

Several simplifications to the experimental model were made to simplify the meshing process as well as limit the computational resources necessary to obtain solutions. A typical mesh consists of approximately 37 million cells, which is about the threshold of the computational resources currently available to the project. The changes to the model can be seen in Fig. 4. The engine pylon has been removed as it creates several issues in meshing, and has little aerodynamic effect to the contribution of lift to this model. The fuselage blend has also been removed as its contribution to the wing's aerodynamic effects is negligible ${ }^{8}$. It will be seen that the first step in the investigation was eliminating the engine as a potential cause of the oscillatory behavior.

Figure 4. Simplified geometry for computational modeling of AMELIA.

Figure 5 shows a planform view of the computational geometry, for this study, defining three spanwise reference planes. The planes are chosen at locations of $x=0.48 \mathrm{~m}$ (inboard of the engine), $\mathrm{x}=0.55 \mathrm{~m}$ (located on the engine 
centerline), and $\mathrm{x}=1.28 \mathrm{~m}$ (arbitrary outboard wing location). These planes will be used for visualizing the flow field henceforth.

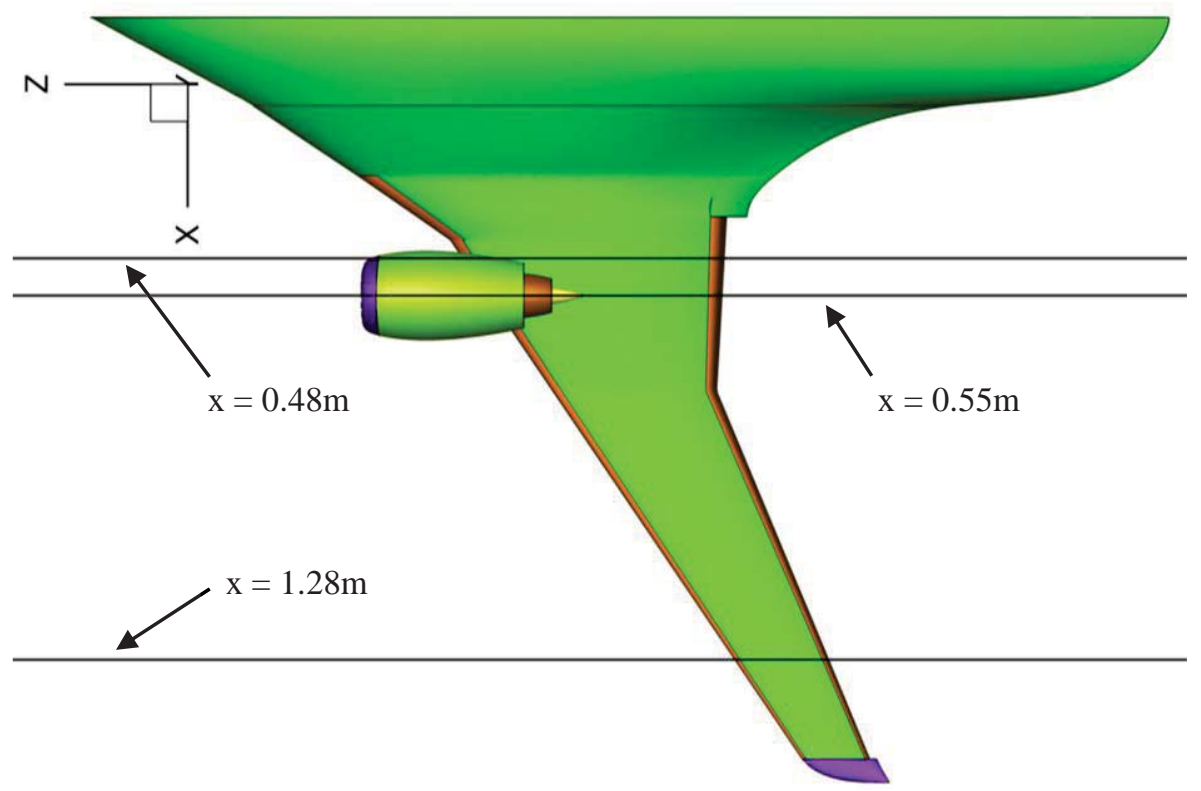

Figure 5. AMELIA planform view showing reference planes.

\section{Unsteady Force Monitor Definition}

Oscillatory behavior has been observed in the lift coefficient as it is tracked per iteration during steady state CFD simulations. Figure 6 shows the oscillatory behavior in the lift coefficient as a function of iteration number. The oscillations result in up to a $10 \%$ fluctuation in the total lift coefficient, which does not dampen out as the solution progresses towards steady state. Similar trends can be seen in the drag coefficient, although the pattern is not as periodic, and will therefore not be discussed. In the flow field, the oscillations are visualized as a confined region of flow on the trailing edge of the inboard wing. It shows a repetitive cycle of separation and subsequent reattachment with a period on the order of 200 iterations for the case with the engine present in the simulation. The lift coefficient is at a maximum (peak) point, labeled in Fig. 6, when the flow is attached over the trailing edge inboard wing section, and at a minimum (trough) point when the flow separates.

The differences in the minimum and maximum points can be seen in Fig. 7, showing pressure coefficient over the upper surface of the

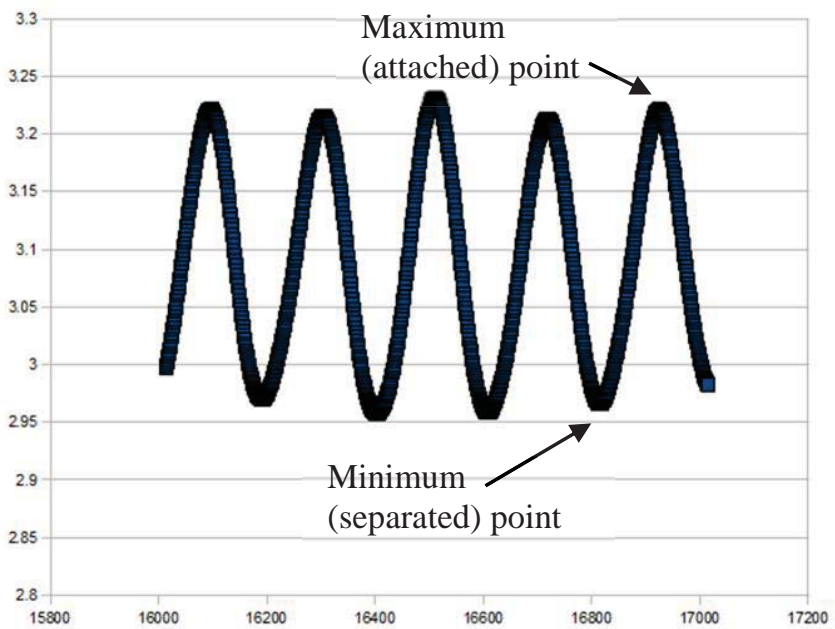

Figure 6. Oscillation in the lift coefficient as a function of iteration.

wing at $x=0.48 \mathrm{~m}$. As seen in the figure, the pressure coefficient from the peak and trough in the oscillation varies drastically, but only at the trailing edge (i.e. from $\sim 0.8 \leq \mathrm{z} / \mathrm{c} \leq 1.0$ ). Figure 8 plots the outboard pressure coefficient distribution for the same two iterations at $x=1.28 \mathrm{~m}$. The figure shows that the pressure coefficients at the points of interest are nearly identical. Thus, it is believed that the solution is converged throughout the entire domain except for the unsteadiness occurring on the trailing edge of the inboard wing. This "steady state" oscillatory condition will henceforth be referred to as fully developed periodic flow. 


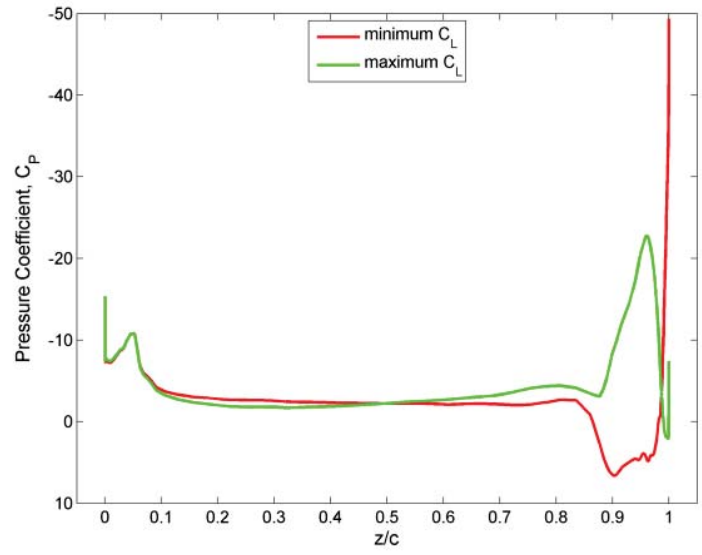

Figure 7. Chordwise pressure coefficient for the peak Figure 8. Chordwise pressure coefficient for the peak and trough in the oscillation at $x=0.48 \mathrm{~m}$.

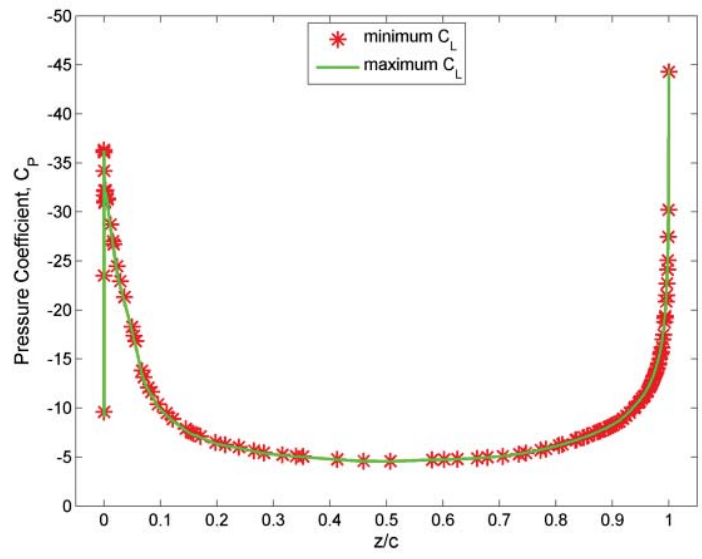
and trough in the oscillation at $x=1.28 \mathrm{~m}$.

The fully developed periodic flow can be better visualized with contours of Mach number, presented in Fig. 9. For the attached flow, the separated region has reattached, and the pocket of high velocity air has moved upstream to the point where the separation initially occurred. The maximum Mach number at the slot exit decreased from 1.19 to 1.09 going from separated to attached, respectively. It should be noted that the flow remains attached to the Coanda surface throughout the solution process, further indicating this phenomenon is not associated with what is in the literature.

Figure 9. Contours of Mach number at $\mathrm{x}=\mathbf{0 . 4 8 m}$.

Another useful quantity to defining separation is surface pressure. Figure 10 provides surface pressure contours for 7 different iterations in the solution, as given by the force monitor plot in the bottom right corner of the figure. The time span of the plots is over 1.5 periods of oscillation. It is seen that the separation emanates from the slot and "washes upstream". Note the sinusoid-like humps from which the "wash-out" originates. 


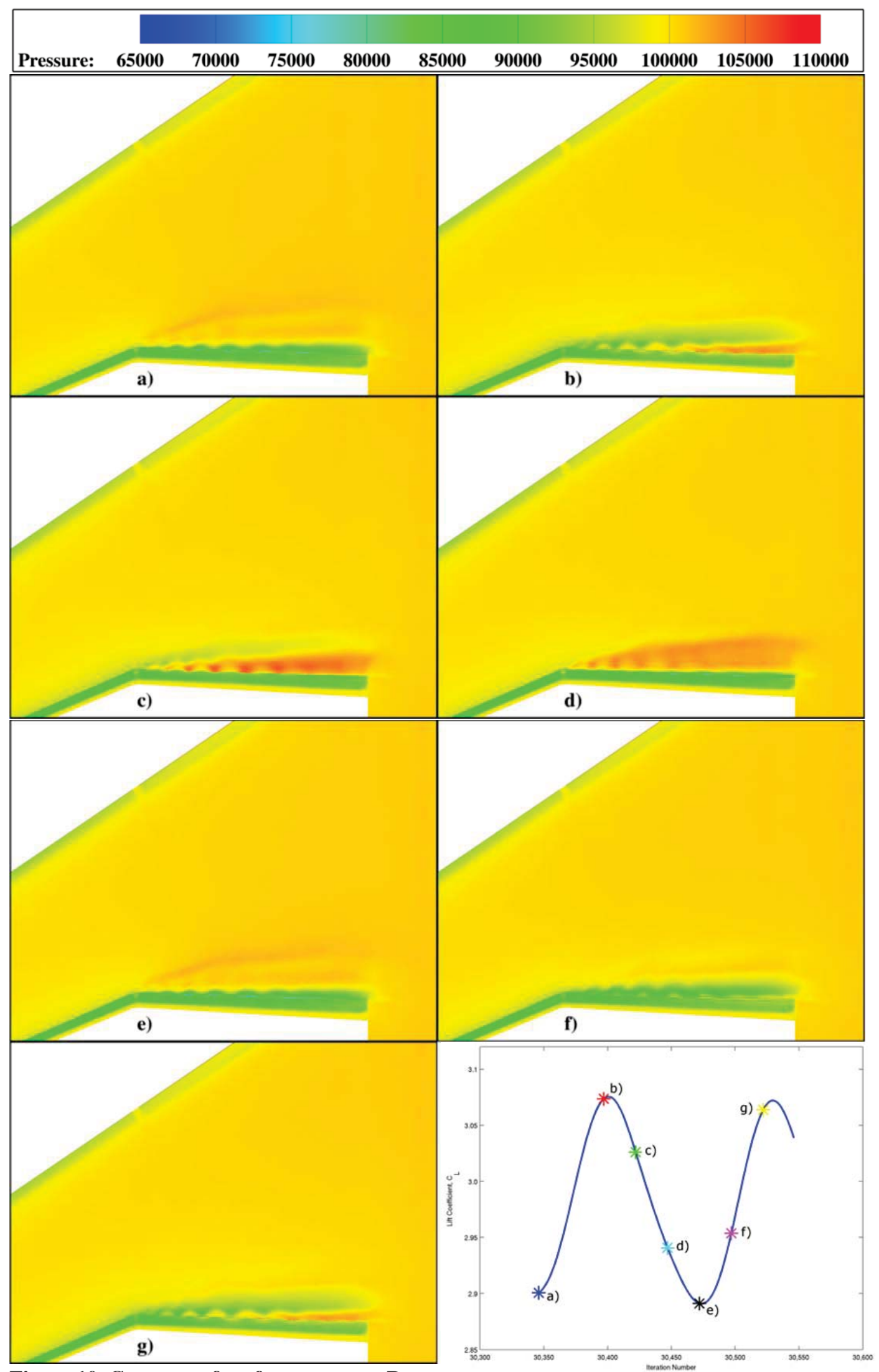

Figure 10. Contours of surface pressure, $\mathrm{Pa}$.

American Institute of Aeronautics and Astronautics 


\section{Computational Grid Generation}

The accuracy of any CFD solution is directly related to the quality of the computational grid, thus adequate time and resources must be dedicated to ensuring a quality grid. The quality of the grid can have significant implications on the stability and convergence of the numerical scheme and accuracy of the results. All of the meshes are hybrid grids, taking advantage of both structured and unstructured cells. An unstructured triangle surface mesh is built up, giving way to unstructured tetrahedral volume elements. Boundary layer effects are captured with prism cells built up to yield a $\mathrm{y}^{+}$as close to unity as computationally possible. The far-field boundaries are meshed with structured hexahedral elements.

While unstructured meshes are ideal for complex geometries, they are not ideal for complex flow physics. In an attempt to better capture the complex flow physics of AMELIA, Cal Poly is currently working on structured grid generation. Pham ${ }^{11}$ provides the details of the progress thus far. The structured mesh generation allows for increased accuracy in the CFD solution, as well as more efficient occupation of the volume, resulting in significantly fewer cells, allowing for further refinement than with the unstructured cells.

\section{A. Unstructured Surface Mesh}

Unstructured surface grids were chosen for their ease and speed of generation while retaining relatively high quality. Figure 11 and 12 show front and back views of the unstructured surface mesh, respectively. Adequate refinement of areas of high curvature, and high flow gradients is necessary. A closer view of the surface mesh near the trailing edge slot can be seen in Fig. 13. As shown, there is adequate refining of cells in the slot and flapped regions.

Figure 11. Front view of the unstructured triangle surface mesh of the computational geometry.

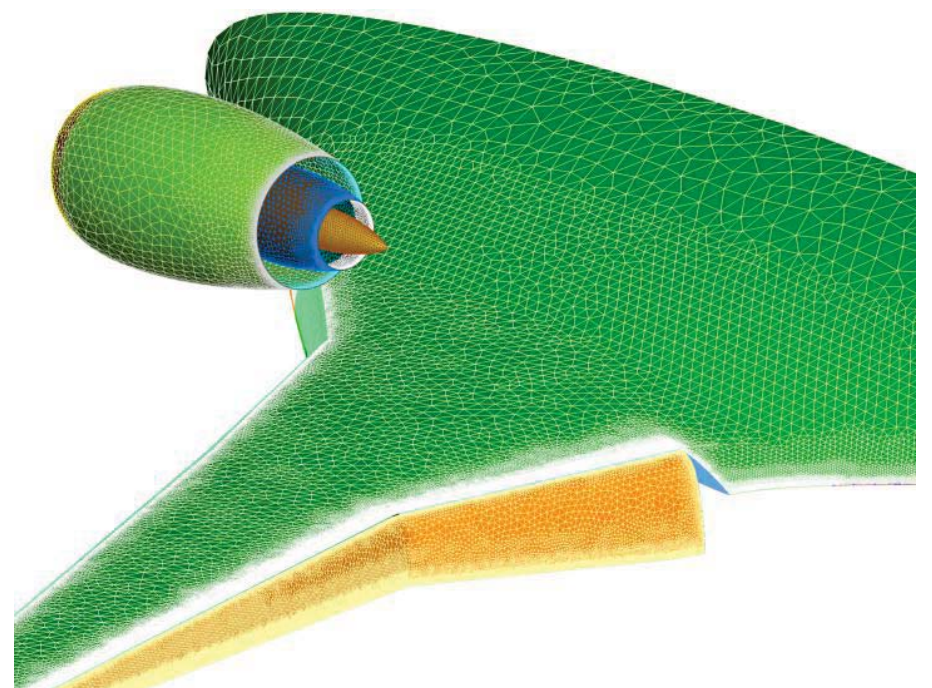

Figure 12. Back view of the unstructured triangle surface mesh of the computational geometry.
Figure 13. Trailing edge inboard slot surface mesh detailed view. 


\section{B. Unstructured Volume Mesh}

The choice of unstructured volume mesh was largely driven by the automated meshing features in ICEM CFD ${ }^{12}$. The tetrahedral elements allow for sufficient mapping around complex geometries, like those associated with AMELIA. The volume elements were grown using the Octree algorithm in ICEM CFD. This algorithm uses a topdown approach where the outer domain is meshed first before resolving the surface of the geometry. This method ensures refinement at the surface to a tolerance, but maintains larger cells wherever possible. The mesh quality inside the slot and plenum become especially important to the quality of the solution, as well as sufficiently refining regions near the engine exhaust and flapped surfaces. Figure 14 shows a cross section view of the volume mesh using the Octree algorithm.

An alternate meshing approach was used for several solutions using the Delaunay algorithm, also available in ICEM CFD. The Delaunay method differs from the Octree method in that it is a bottom-up method. The elements are grown out from a pre-existing surface mesh. The result of this algorithm is a more efficient use of space (reduction in cell count of roughly 5 million cells) and smoother transitions. Figure 15 shows a cut plane of the mesh using the Delaunay method. To better capture the wake, the downstream flap region was refined. It should be noted that changing the cell resulted in no change to the development of fully developed periodic flow.

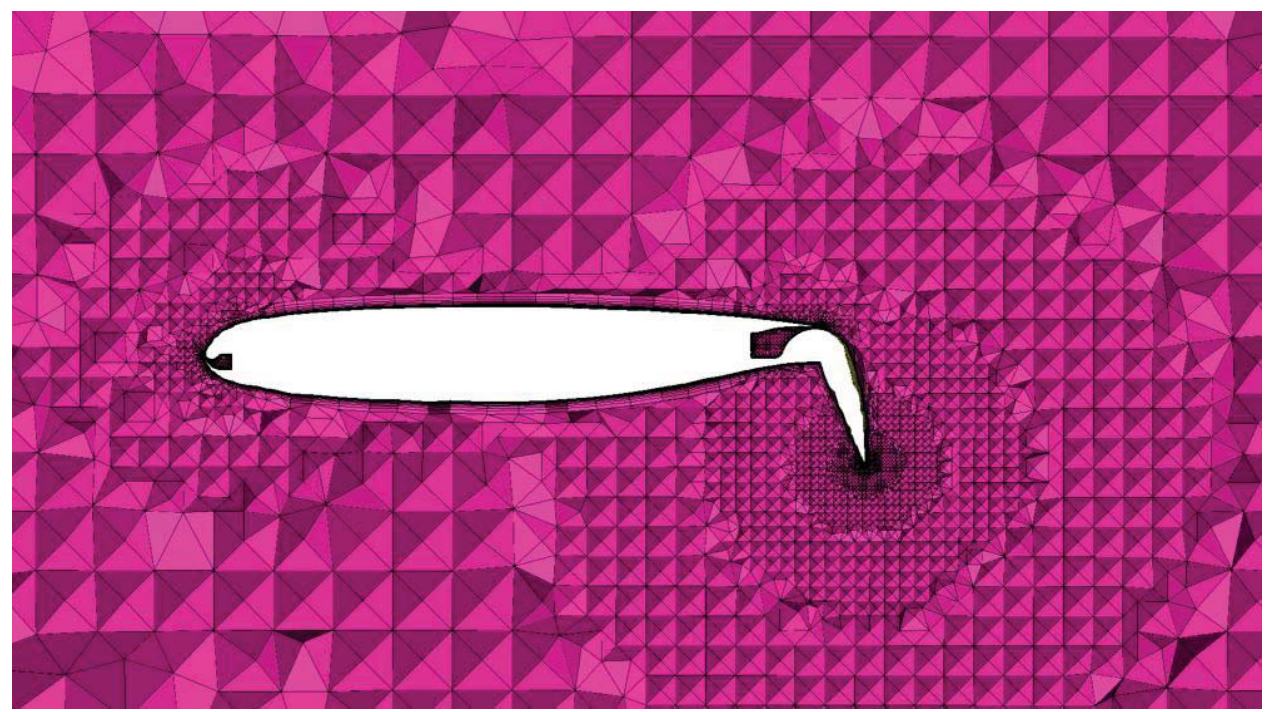

Figure 14. Cross sectional view of the unstructured volume mesh using the Octree algorithm.

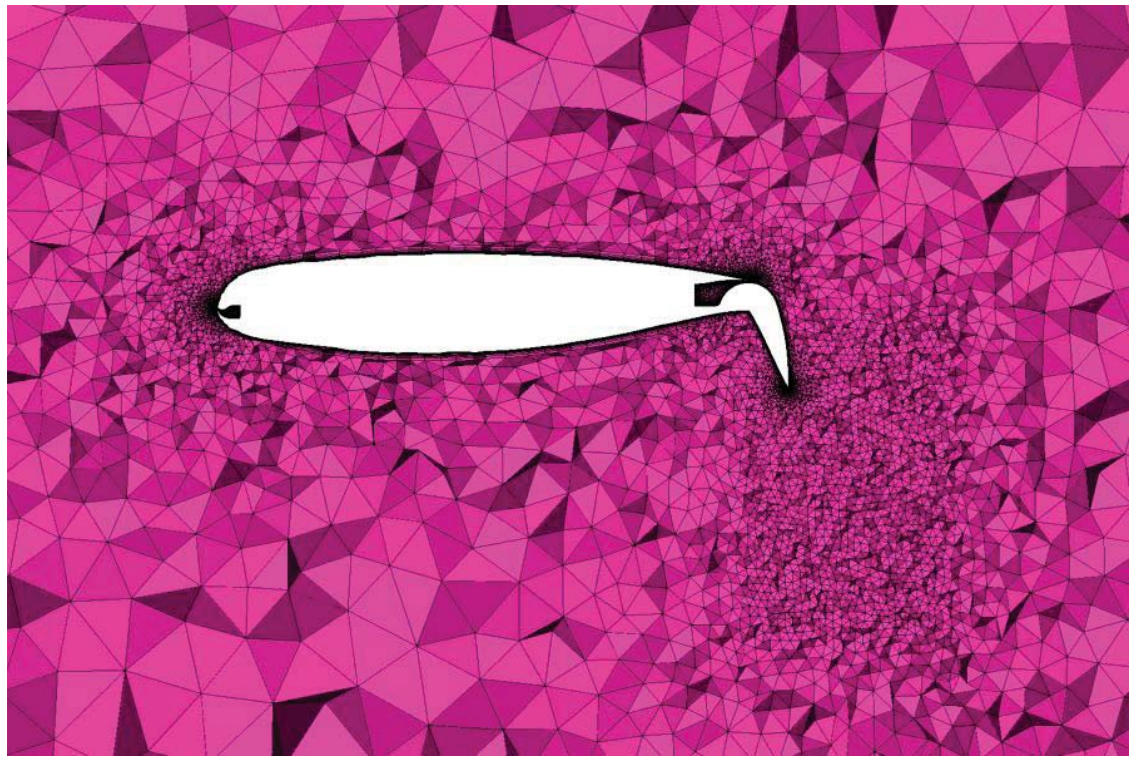

Figure 15. Cross sectional view of the unstructured volume mesh using the Delaunay method.

American Institute of Aeronautics and Astronautics 


\section{Boundary Layer Refinement}

Near wall modeling is especially important for AMELIA. The interactions of the high speed jet flow, boundary layer, and freestream flow all occur near the wall and adequate refinement is necessary to capture these effects and obtain a quality solution. Ideally, the mesh would have $\mathrm{y}^{+}$of one everywhere, with a growth ratio away from the wall of 1.15 to 1.20 , obtaining at least 10 cells within the boundary layer. This is, however, not always feasible for the computational resources available. Thus, the prism layers are grown with an inflation rate of around $40 \%$ to yield an acceptable transition between prisms and tetrahedral elements. As will be discussed later, the boundary layer height scales with growth ratio and could be a factor in causing the oscillations. Figure 16 shows a region of near wall mesh resolution showing the prismatic elements and transition to tetrahedron, on the wing upper surface. This transition cannot be held everywhere, however that is the intent.

Figure 16. Prism layer transition to unstructured tetrahedron on the wing upper surface.

\section{Results and Discussion}

This section will summarize the investigative effort thus far in determining the source of the unsteady behavior in the lift coefficient. As there could be a potentially endless list of possible sources, an argument will be made for each of the cases presented, and its significance to this effort.

For each case presented, the angle of attack is zero, the thrust coefficient (where applicable) is 0.8 and the momentum coefficient is 0.3 . Pham ${ }^{11}$ defines the thrust and momentum coefficient as well as other relevant solver boundary conditions. The cases are all solved with the commercial code FLUENT ${ }^{13}$. Within FLUENT, the implicit density based solver is used with the $k$ - $\omega S S T$ turbulence model. The Courant number for the cases is held between 4 and 6.

\section{A. Engine Effects}

The first step in this investigation was eliminating engine effects from the source of the unsteadiness. The engine entrainment work (done prior to this) was based largely on coupling the CCW and upper surface blowing (produced by an above the wing mounted engine) in order to entrain engine flow and increase lift. Modeling the geometry without the floating engine shows that the flow becomes fully developed and periodic. This is shown in Fig. 17. Being able to decouple the engine effects from the case decreases the overall complexity of the CFD solution. Note the solution was carried out to more than 31,000 iterations; the periodicity in the flow remains and isn't damped. Comparing to Fig. 6, the magnitude of the lift coefficient has decreased due to the loss of exhaust entrainment. The amplitude of oscillation has, however, remained about the same. As the solution proceeds, the only apparent change is a decrease in the period of oscillation to approximately 100 iterations. Recall the period for the engine solution at 18,000 iterations was about 200 iterations. It should be noted that at 18,000 iterations in the no engine solution, the period is nearly identical at 200 iterations. 
Figure 17. Lift coefficient force monitor without the engine.

\section{B. Viscous Effects}

To simplify the flow and possibly eliminate the mesh itself as the potential culprit, an inviscid case was run. Turning off viscous effects eliminates the development of the boundary layer and presents a "powered lift only" solution. This "powered lift only" solution eliminates the flow interactions between the boundary layer and the Coanda flow over the wing surface, which in and of itself could prove very complex. In developing a mesh, simply having the first point from the wall within a $\mathrm{y}^{+}$of unity is not the entire story. The growth ratio of the prismatic cells that capture the boundary layer can also prove just as important. Stretching cells too fast can generate boundary layers that are thicker than is appropriate ${ }^{\ddagger}$. And as thicker boundary layers separate easier than thin boundary layers, this could cause separation off the trailing edge.

This seems plausible as the local Reynolds number at the trailing edge of the inboard wing is also significantly higher than the outboard wing.

Figure 18 presents the lift force monitor for the inviscid case. It can be deduced that the period flow does not develop. Because the flow does not become periodic the mesh cannot be ruled out as the cause of the oscillations. As mentioned, this case only rules out the "powered lift only" effect. It does not eliminate boundary layer effects (that may or may not be coupled with powered lift) or any possible unsteady vortex shedding (to be discussed in the next section) that could occur due to the interactions of the high and low speed flows.

Note that it is not important to drive this solution to a true steady state value. A typical solution is iterated until it develops periodicity, at which point it is said to be "converged". Here though, the force monitor history does not show the oscillatory behavior that has been observed below 20,000 iterations for each of the cases presented thus far, at which point it was deduced that the behavior would not develop.

Figure 18. Lift coefficient force monitor for a inviscid solution.

\section{Vortex Shedding}

It has been shown that the area of interest is inclusive to the trailing edge inboard wing location. It was also discussed and shown in Fig. 10 that the separation seems to emanate from the trailing edge slots and wash-out upstream. Taking this into consideration, as well as there being a blunt lip at the trailing edge just above the slot, the authors hypothesized that the separation could be induced by an unsteady vortex shedding phenomenon, much like flow over a backward facing step or a cylinder. For the cylinder, the vortex shedding instability creates unsteadiness in the forces acting on the cylinder, hence unstable force monitors. For the trailing edge region, the flow over the upper surface of the wing has significantly lower velocity than the jet flow coming out of the slot. Much like a backward facing step, this could cause a recirculation region that could potentially propagate upstream of the lip and

${ }^{\ddagger}$ Private communication with Dr. Kyle Anderson at University of Tennessee at Chattanooga 
cause separation. The buildup of high momentum flow from the leading edge slots would be the enabler for reattachment and the cycle is formed. To mitigate this "shedding" effect, a sharp lip was put in place of the blunt lip that is on the model. Figure 19 shows the difference in the blunt and sharp lip, at the trailing edge slot.

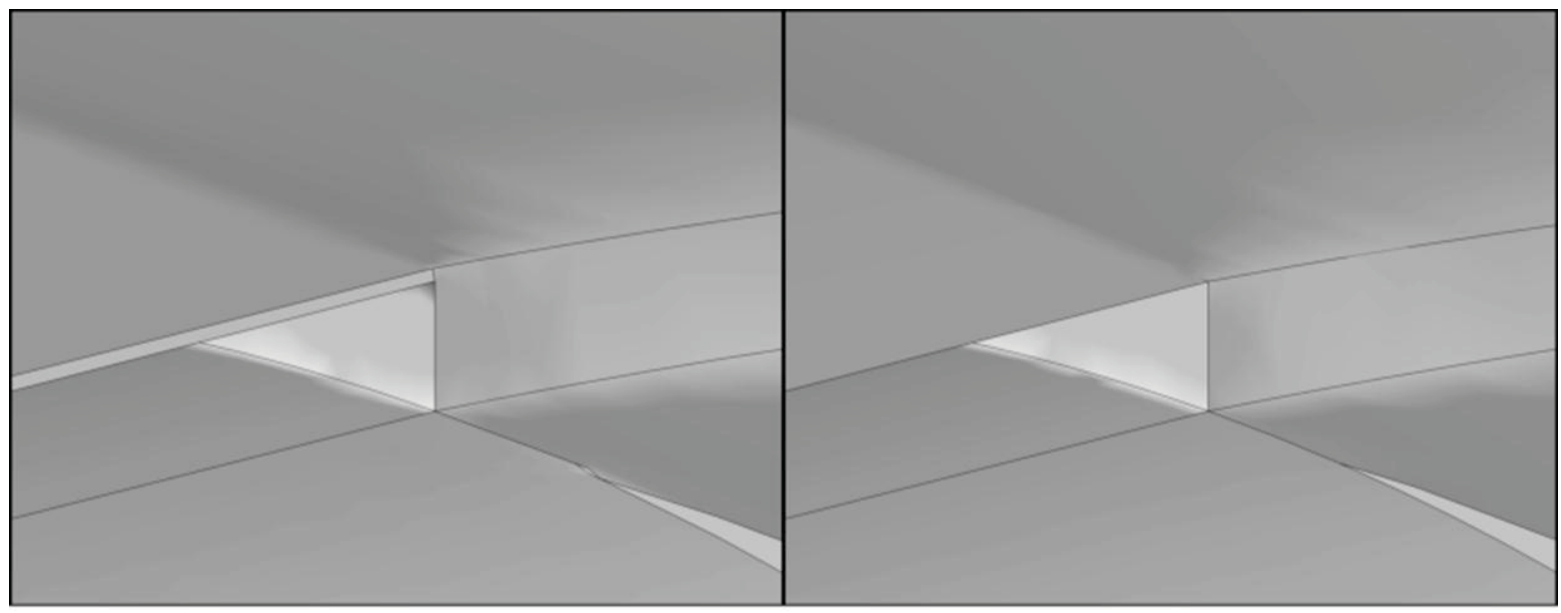

a) Blunt Lip

b) Sharp Lip

Figure 19. Trailing edge slot geometry showing blunt and sharp lip.

Figure 20 presents the force monitor for the sharp trailing edge lip. As seen, the fully developed periodic flow does occur. It should be noted, that the amplitude has decreased significantly to about $2 \%$, an encouraging result. The period of oscillation has also increased from the baseline case with the engine, to approximately 100 iterations.

Upon seeing that the fully developed periodic flow still occurred, several other reasons were identified. Like the flow over a cylinder, vortex shedding (visualized by low velocity and high pressure regions) is easily visualized with a contour plot of either pressure or velocity. Looking at those contours for any of the solutions presented, there are no signs of vortex shedding, or at least none of the magnitude that could cause a $10 \%$ fluctuation in the lift coefficient. The other factor was the sheer magnitude of the blunt lip. On the wind tunnel and computational models, the slot height reaches ten thousandths of an inch (about the thickness of 3 pieces of paper stacked) in its smallest location. As seen in Fig. 19 , the lip is significantly smaller than the slot height. Based on this, it is unlikely that a vortex shed from something that is less than three pieces of paper thick could cause 10\%

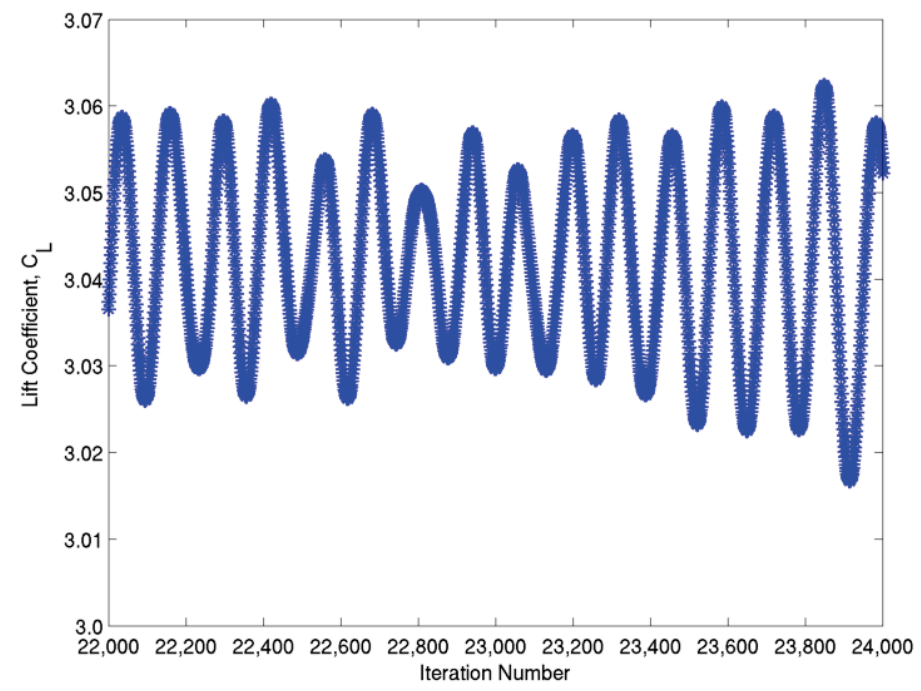

Figure 20. Lift coefficient force monitor for sharp trailing edge lip geometry. fluctuations in the lift coefficient.

Another area of interest where vortex shedding or shear layer instabilities could cause interesting flow physics is the leading edge of the wing. About half-way across the wing there is a region on the leading edge where there is no blowing (where the two leading edge plenums are separated, see Fig. 3). The interaction between the near sonic flow from the leading edge slot and the near stagnant flow, where there is no blowing, could create a "sonic sandwich" of interesting and complex flow physics. Due to the magnitude of these gaps of non-blown regions, it does not seem likely as the source of the separation. However, it is not well understood by the authors at this time, the implications that this "sonic sandwich" may have on the trailing edge separation. 


\section{Wing Cross-flow}

One of the conditions of the flow leaving the slot is that the direction of travel is normal to the plane of the slot. This constrains the circulation control jet to flow across the wing at angle that is perpendicular to the leading edge. For a single section, linearly tapered wing, this does not present any significant problems. However, the AMELIA wing has two sections of differing taper. The taper ratio of the outboard wing section is very large, so the sweep of the leading and trailing edge is nearly the same, accordingly the leading edge flow can move over the wing and down the slot. However, at the inboard section, there is essentially no sweep at the trailing edge, and the flow must turn significantly to meet normal to the trailing edge slot. This turning, coupled with the shearing of the freestream and leading edge slot flows, creates a significant cross-flow component over the wing that is believed to create separation. Figure 21 shows two sets of streamlines originating from the leading edge plenum, over the inboard and outboard wing sections. The outboard streamlines are very uniform and do not require any turning to meet normally at the trailing edge slot. The inboard streamlines "fan out" indicating cross-flow and turn to meet normal with the trailing edge at the same region where separation occurs. It is the authors' opinion that this reasoning best explains the centralized location of the separation that is causing the oscillations.

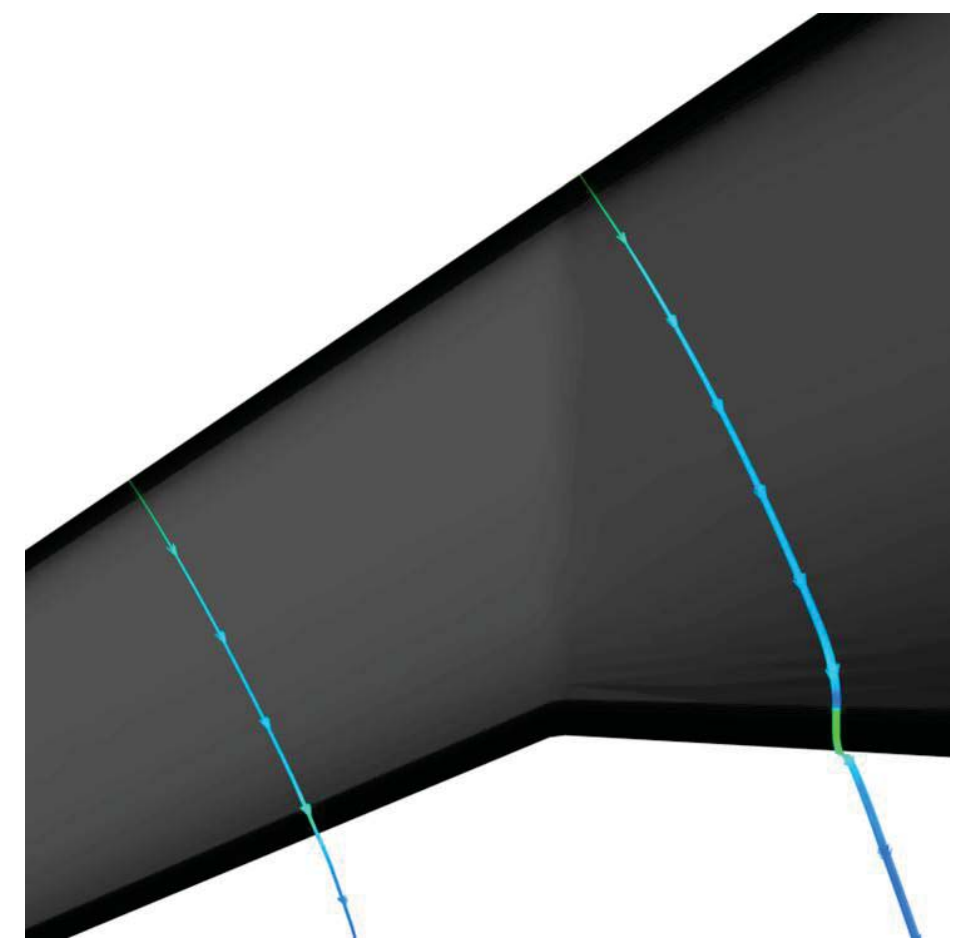

Figure 21. Streamlines over wing upper surface indicating cross-flow at inboard section.

\section{Conclusions and Future Work}

Oscillations are observed in the lift coefficient, of up to $10 \%$, as it is tracked per iteration for steady state CFD simulations. These oscillations were shown to be due to viscous effects and do not dampen as the solution proceeds through steady state. Although the cause of the unsteadiness has not been identified, likely causes have been investigated and some eliminated as the potential source of this behavior. Identifying sources of the unsteadiness are difficult to pinpoint as this seems to be a rather abstract occurrence. Foremost, the engine effects were eliminated as a source. This allowed for a simplified computational model that reduced both the solving complexity and mesh size. Initially, a strong case was made for vortex shedding off of the blunt lip of the trailing edge slot, like the case with a backward facing step or a cylinder. Although the amplitude of the oscillations was reduced, this argument does not hold due to the sheer size of the model. Vortex shedding, that causes $10 \%$ fluctuations in lift, does not seem plausible at the lip because the slot height itself is only ten thousandths of an inch at its smallest location. At this point, it is proposed that cross-flow induces separation over the trailing edge of the inboard wing section due to the 
flow turning to meet the flow normal condition at the slots. Assuming this to be the case, it is unknown why the periodic flow did not develop for the inviscid solution.

In the future, work will continue into finding the origin of the unsteady oscillatory behavior in the lift force monitor. The wing will be run without blowing with flaps and slots retracted, representative of a "clean" wing configuration. This solution will determine if the oscillations are due to powered lift effects, and not due to the wing design. Note it is not the purpose of the grant to design a wing to meet performance or aerodynamic goals, but to obtain data for validation of predictive models. Oscillations occurring due to the aerodynamics of the wing (i.e. due to the design), are consequently out of the scope of this work. The implications of the "sonic sandwich" at the leading edge will also be examined.

Once the source of the unsteadiness has been identified, it will need to be mitigated. As this could still be a physical phenomenon, the importance in finding the cause is increasingly important as the wind tunnel test date approaches.

\section{Acknowledgments}

This work was funded as a part of a NASA Research Announcement award under Contract \# NNL07AA55C with Craig Hange and Clif Horne as technical monitors. The authors would like to personally thank Dr. Dave Whitfield and Dr. Kyle Anderson at the University of Tennessee at Chattanooga, as their discussions helped to shape this work. The authors also wish to thank Bryan Blessing, Eric Paciano, John Pham, Rory Golden, and Robert Ehrmann as their expertise has been invaluable to the development of this work.

\section{References}

\footnotetext{
${ }^{1}$ Abramson, J., and Rogers, D. W., "High-Speed Characteristics of Circulation Control Airfoils," AIAA-83-0265, Jan., 1983.

${ }^{2}$ Wood, N., "Circulation Control Airfoils Past, Present, Future," AIAA-85-0204, Jan., 1985.

${ }^{3}$ Liu, Y., "Numerical Simulations of the Aerodynamic Characteristics of Circulation Control Wing Sections," Ph.D. Dissertation, School of Aerospace Engineering, Georgia Institute of Technology, Atlanta, Ga, 2003.

${ }^{4}$ Marshall, D. D., and Jameson, K. K., "Overview of Recent Circulation Control Modeling Activities at Cal Poly," $48^{\text {th }}$ AIAA Aerospace Sciences Meeting and Exhibit, AIAA, Orlando, Fl, Jan., 2010, AIAA-2010-348.

${ }^{5}$ Blessing, B. H., Pham, J., and Marshall, D. D., "Using CFD as a Design Tool on New Innovative Airliner Configurations," $47^{\text {th }}$ AIAA Aerospace Sciences Meeting and Exhibit, AIAA, Orlando, Fl, Jan., 2009, AIAA-2009-0045.

${ }^{6}$ Englar, R. J., "Development of Circulation Control Technology for Powered-Lift STOL Aircraft," Lockheed Georgia Company, May, 1987.

${ }^{7}$ Golden, R., and Marshall, D. D., "Design and Performance of Circulation Control Flap Systems," $48^{\text {th }}$ AIAA Aerospace Sciences Meeting and Exhibit, AIAA, Orlando, Fl, Jan., 2010, AIAA-2010-1053.

${ }^{8}$ Blessing, B. H., "Analyzing the Engine Entrainment of an Over the Wing Engine Coupled With a Circulation Control Wing," International Powered Lift Conference, Philadelphia, Pa, October, 2010.

${ }^{9}$ Jameson, K. K., et al., "The Wind Tunnel Model Design and Fabrication of Cal Poly's AMELIA 10 Foot Span Hybrid Wing-Body Low Noise CESTOL Aircraft, Part I," $49^{\text {th }}$ AIAA Aerospace Sciences Meeting and Exhibit, AIAA, Reston, VA (submitted for publication).

${ }^{10}$ Jameson, K. K., et al., "Part II: Preparation for Wind Tunnel Model Testing and Verification of Cal Poly's AMELIA 10 Foot Span Hybrid Wing-Body Low Noise CESTOL Aircraft," $49^{\text {th }}$ AIAA Aerospace Sciences Meeting and Exhibit, Reston, VA (submitted for publication).

${ }^{11}$ Pham, J., "Predictive Modeling using CFD on a Circulation Control Aircraft for Wind Tunnel Validation," $49^{\text {th }}$ AIAA Aerospace Sciences Meeting and Exhibit, AIAA, Reston, VA (submitted for publication).

${ }^{12}$ ANSYS ICEM CFD, Software Package, Ver. 12.1, ANSYS, Inc., Canonsburg, PA, 2009.

${ }^{13}$ ANSYS FLUENT, Software Package, Ver. 12.1, ANSYS, Inc., Canonsburg, PA, 2009.
} 